urine was moderately coagulable, and below the normal quantity; its specific gravity, 1020 . (Six leeches to the præcordia ordered; two grains of calomel twice a day, and a saline mixture, containing squill and digitalis.) She continued this plan of treatment until the $28 \mathrm{th}$, when the præcordial pain had subsided. The heart's movements were lessened in force and frequency. The urine was copious, feebly coagulable, and its density, 1014. During convalescence, the anasarca disappeared, but the patient remained in a very anomic condition, the urine increasing in coagulability until the 6th of December. From that date the coagulability became less, and speedily disappeared altogether.

(To be continued.)

\section{ON THE DIAGNOSIS OF THE DIFFERENT KINDS OF MUSCULAR PARALYSIS.}

By MARSHALL HALL, M.D., F.R.S., \&e.

Ter kinds of muscular paralysis, of which I propose in this paper to trace the diagnosis, are two-1, the cerebral; 2 , the spinal.

I designate that cerebral paralysis, in which, by whatever means or disease, the influence of the brain is severed; and I designate that form of paralysis spinal, in which, from whatever cause, the influence of the spinal marrow is severed.

It is unnecessary to state, that in the latter case the influence of the cerebrum is removed, as well as that of the spinal marrow.

Before I proceed, I must take the opportunity of briefly alluding to the functions observed in the muscular system, for it is these that are variously affected in paralysis.

The first function of the muscular system which I shall mention is voluntary motion, the effect of volition; the second function, or rather affection of the muscular system, is the effect of emotion; the third is reflex action; the fourth is the tone of the muscular system; the fifth is the irritabitity of the muscular fibre. All these are wonderfully involved in every muscular act or action, though one may be principally so, and the affection of each becomes to the physiologist the source of diagnosis in cases of paralysis; for I may take this opportunity of stating, that-

In the diseases of the nervous system, the physiology is the diagnosis.

I will briefly detail the particulars of two cases, which may be taken as types of the rest.

The first was a case of hemiplegia, affecting the right side. The muscles of the right arm were paralytic, atrophied, and contracted, and rigid. We placed both hands in one basin, containing water with a little salt, and a foot in another, and we passed a galvanic current through the limbs so disposed, beginning with the smallest force which would produce an obvious movement. Invariably, in many trials, the paralytic arm was moved by the slighter force, and the most by a greater force, whatever the direction of the current.

The second case was that of a little girl, aged two years and $\mathbf{a}$ half, in whom the left arm had been suddenly affected with complete paralysis nearly two years before, the head having at the time of the seizure been drawn to one side, and the child being under the influence of dentition. In this case the para. lysis of muscular power was, as I have stated, complete; the arm was atrophied, the muscles flaccid, the head moving on the wrist, if held in the dependent position, absolutely uncontrolled by muscular influence. We applied the galvanic test as before, using the mildest force which would produce an obvious effect. Now, the unaffected $\operatorname{limb}$ was first and most affected by the galvanism!

In these two cases we have the marked forms of cerebral and spinal paralysis respectively; in the first, we observe a very marked tone (contraction); in the second, abolition of tone, (flaccidity); in the first, we have augmented, in the second, diminished, irritability of the muscular fibre. In the first, too, there was agitation of the arm on any emotion; in the second, none. I had no satisfactory mode of testing the existence or absence of reflex action.

The physiologist, and now, I think, the practitioner, will see the value of these phenomena to science, and in their translation into diagnosis.

In hemiplegia, the seat of motion, the source of tone, the source of irritability, are left; in spinal paralysis, the seat of emotion, the source of the tone of the muscles, and of the irritability of the muscular fibre, are removed! It must be remembered, that in both cases the miscular mass was equally atrophied. What are the obvious physiological inferences from these facts, and what are their practical advantages in a diagnostic point of view? I leave the replies to these quess tions to my readers, observing, that the first must be diametrically opposed to the opinions recently advocated by the late Dr. J. Reid, by Dr. Carpenter, Dr. Todd, \&c.

The cases themselves, with many others, have all been witnessed by my friend, Mr. Henry Smith, of Torrington-square, and so frequently, and so carefully, that they may, I believe, be received as accurately ascertained facts, trom. which equally cautious inferences may be fearlessly drawn.

Are not these inferences-first, that the cerebrum is the seat of volition; second, that a lower portion of the encephalon (the medulla oblongata?) is the seat of emotion; third, that the medulla spinalis is the seat or source of tone or tonicity in the muscular system? and fourth, of the irritability of the muscular fibre ? and fifth, that the two last are not dependent nutrition merely?

Will not my readers pardon a little enthusiasm in the view I take of the value of these investigations, and a little indignation, not unmingled with contempt, at the treatment they have received at the hands of incapable opponents, at the Royal Society, and elsewhere? Can any ingenuous person read this simple narrative without deploring that odium Physiologicum which has met or persecuted this inquiry at every step?

I may here add that the cause of cerebral paralysis is chiefly, the injured substance of the cerebrum; whilst spinal paralysis may arise from the injured substance of the spinal marrow, or from the injured substance or function of spinal nerves - any disease, in effect, which severs the infuence of the spinal marrow from the muscles. In this sense the paralysis. arising from lead is -spinal paralysis. It is well known that in this disease the tone of the muscles and the irritability of the muscular fibre are diminished.

Of paroxysmal paralysis, and of algo- and spasmo-paralysis, I have treated in recent papers in THE LANCET, to which I beg leave to refer my reader. I may observe here, that the hand and arm contracted by tone in cerebral paralysis are contracted symmetrically, and without distortion; all the muscles being equally affected: the flexars overcome the extensors, the arm is bent, and the haud closed firmly but regularly. In spasmo-paralysis, certain muscles only are excited to contraction, and that contraction is irregular, clonic, and tonic, and the arm and hand are affected with various defor. mities, scarcely admitting of description, but which it would be highly interesting to depict.

Manchester-square, August, 1849.

Postscript; (being a Note on the difference between Tetanus and Hydrophobia.)

Hydrophobia, like the effects of strychnine, is homatogenic. The spinal marrow is in a state of abnormal and extreme excitability or erethism. But, as there is no permanent excitant, there is no spasm, except under the influence of an occasional one. There is perfect intermission of all spasmodic affection in the intervals between the paroxysms.

Tetanus, on the contrary, is neurogenic; and whilst the spinal marrow is equally in a state of undue excitability, there is a constant source of excitation in the injured, irritated nerve. There is, therefore, constant spasm, and remission only, in the intervals between the paroxysms-that is, in the absence of occasional excitants,-a paroxysmal exacerbation of spasm taking place when these are in operation.

Such is the grand pathological distinction between these diseases. I may make an additional remark: if in a case of tetanus the injured nerve was divided, the remittence of spasm would become intermittence. This view suggests the real value of amputation in such cases, discussed by Larrey and by Dupuytren, the former recommending, the latter dissuading from, this measure.

I may add, that hydrophobia having its source in the blood, its symptoms are uniform; there is always and equally laryngisnus; whereas the symptoms of tetanus vary with the special nerve injured, and there is sometimes laryngismus, sometimes pharyngismus, sometimes not, as there is, in different instances, emprosthotonus, episthotonus, pleurosthotonus.

There is, further, the interesting question relative to tracheotomy in these diseases, and the not less interesting question, in a scientific point of view, of the morbid anatomy; with the latter object, $I$ have devised an instrument for readily opening the cranium and spinal canal.

My reader will not be surprised, that with all these questions in my mind, I should meditate a journey as far as the seat of the Austrian and Hungarian warfare, with the view of observing tetanus in the wounded of those battle-fields. 